Apidologie, 1987, 18 (2), 147-162

\title{
DER MASSENWECHSEL DER GRÜNEN TANNENHONIGLAUS CINARA PECTINATAE IN VERSCHIEDEN STARK ERKRANKTEN TANNENBESTÄNDEN 1977-1985
}

\author{
Gerhard LIEBIG \\ Landesanstalt für Bienenkunde, Postfach 700562 \\ D-7000 Stuttgart 70
}

\section{ZUSAMMENFASSUNG}

Der Massenwechsel der bienenwirtschaftlich wichtigen Grünen Tannenhoniglaus Cinara pectinatae wurde von 1977-1985 in 7 verschieden stark erkrankten Tannenbeständen in Baden-Württemberg beobachtet. Charakteristisch für den Massenwechsel ist die Vermehrungsphase von Mai bis Juli und der Zusammenbruch im August/September. In den stark erkrankten Tannenbeständen weicht der Massenwechsel ab : im Sommer bricht die Population regelmäßig bereits im Juni zusammen und es kommt häufig zu einer Spätvermehrung im Herbst.

\section{EINLEITUNG}

Die Imkerei wird in Deutschland wie in vielen Teilen Mitteleuropas hauptsächlich im Nebenerwerb betrieben. In Süddeutschland werden etwa $60 \%$ des Honigs im Wald geerntet und stammen dabei zum größten Teil aus Honigtautrachten von Fichte und Tanne.

Die Waldtracht tritt sehr unregelmäßig auf. Tanne und Fichte honigen selten weit verbreitet. Die Ertragsunterschiede zwischen entfernt voneinander und auch nahe beieinander liegenden Waldgebieten können im gleichen Zeitraum sehr groß sein. An einem Standort setzt nicht jedes Jahr gute Tracht ein. Zwischen guten Waldhonigjahren können 2-10 Jahre liegen.

Die wichtigste - aber nicht die einzige - Voraussetzung für das Einsetzen einer Waldtracht ist der Massenbefall der Honigtauerzeuger. Die Grüne Tannenhoniglaus Cinara pectinatae ist der bienenwirtschaftlich bedeutendste Honigtauerzeuger auf der Weißtanne. Seit 1977 führen wir Untersuchungen 
über die Populationsdynamik von C. pectinatae durch mit dem Ziel zu klären, unter welchen Voraussetzungen es zu einem Massenbefall bzw. zu einer Tannentracht kommt. Bei den Messungen im Freiland wird der Massenwechsel an verschiedenen Standorten über einen Zeitraum von mehreren Jahren verfolgt, um Vergleiche zum Witterungsverlauf zu ermöglichen und außerdem den Einfluß des Standortes sichtbar zu machen.

In den letzten Jahren haben die Waldschäden in der Bundesrepublik Deutschland stark zugenommen. Besonders stark betroffen sind die Tannenbestände des Schwarzwaldes (Schöpfer und HradeTzky, 1985). Da die Tannenerkrankung an den Versuchsstandorten unterschiedlich weit fortgeschritten ist, wird in der vorliegenden Arbeit der Massenwechsel von C. pectinatae in dem Zeitraum 1977-1985 an den untersuchten Standorten vorgestellt und der mögliche Einfluß der Tannenerkrankung diskutiert.

\section{MATERIAL UND METHODIK}

Die phloemsaugende Lachnide $C$. pectinatae besiedelt die Rinde ein- und mehrjähriger Zweige. Sie ist auch bei Massenbefal einzeln sitzend. Aus den überwinterten Eiern schlüpfen im März/April die Stammütter, die sich wie die folgenden Generationen vivipar und parthenogenetisch fortpflanzen. Die ersten $F_{1}$ treten je nach Witterungsverlauf und Höhenlage zwischen Anfang Mai und Anfang Juni auf. Als $F_{4}$ oder $F_{5}$ werden im September die Geschlechtstiere geboren, die sich zu geflügelten Männchen und ungeflügelten Weibchen entwickeln. Nach der Kopulation legen die Weibchen im Oktober/November die Eier einzeln auf den Nadeln ab.

\section{Beschreibung der Standorte (Tab. 1)}

Der Standort 1 in Suttgart-Hohenheim ist eine kleine Anpflanzung von Fichten und Tannen vor dem Gebäude der Landesanstalt für Bienenkunde. Die Bäume wurden 1974 etwa 10-jährig in aufgeschütteten Lehmboden gepflanzt.

Die Standorte 2-8 sind etwa $1 \mathrm{qkm}$ groß und relativ eben, innerhalb der Meßfläche bestehen nur geringe Höhenunterschiede bis höchstens $20 \mathrm{~m}$. Die Standorte 2-7 liegen im Raum südlich von Pforzheim, der Standort 8 im Raum südlich von Freudenstadt. Jeder dieser Standorte wurde unter Berücksichtigung der örtlichen Verhältnisse (Fahrwege, Vorkommen bzw. Anteil von anderen Baumarten, Jungpflanzungen und Kahlflächen) in 3 Meßbereiche unterteilt. Im Herbst 1985 wurden an jedem Standort 10 verstreut stehende Tannen auf ihren prozentualen Nadelverlust bonitiert (SCHRöTER und ALDINGER, 1985). Je nach dem Grad der Entnadelung wurden die Probebäume in die Schadstufen 0 bis 3 eingeteilt. Die Schadstufe 0 bedeutet $0-10 \%$ Nadelverlust, Schadstufe 1 bedeutet 11-25\%, Schadstufe 2 bedeutet 26$60 \%$ und Schadstufe 3 bedeutet mehr als $61 \%$ Nadelverlust. Zusätzlich wurde durch Grobansprache die Schadstufe von Tannen ermittelt, die in unmittelbarer Nähe der Probebäume standen, so daß insgesamt je Standort etwa 100 Tannen nach Schadstufen klassifiziert wurden (Tab. 1).

\section{Beobachtung des Massenwechsels}

Am Standort 1 in Hohenheim wurden jedes Jahr im April an 3 (1977) bzw. 6 (1978-1983) bzw. 8 Tannen (1984 und 1985) je 10 Zweige markiert, von denen jeder mit seinen Verzweigungen etwa $20 \mathrm{~cm}$ breit und etwa $30 \mathrm{~cm}$ lang war (DIN-A-4-Blatt-Größe). Die markierten Zweige befanden sich sowohl an der Peripherie als auch im Inneren des Baumes und waren rundum in Hüft- bis Augenhöhe verteilt. 1985 wurden auch Zweige in der Baumspitze markiert und mit Hilfe einer Stehleiter ausgezählt. Je nach ihrer 


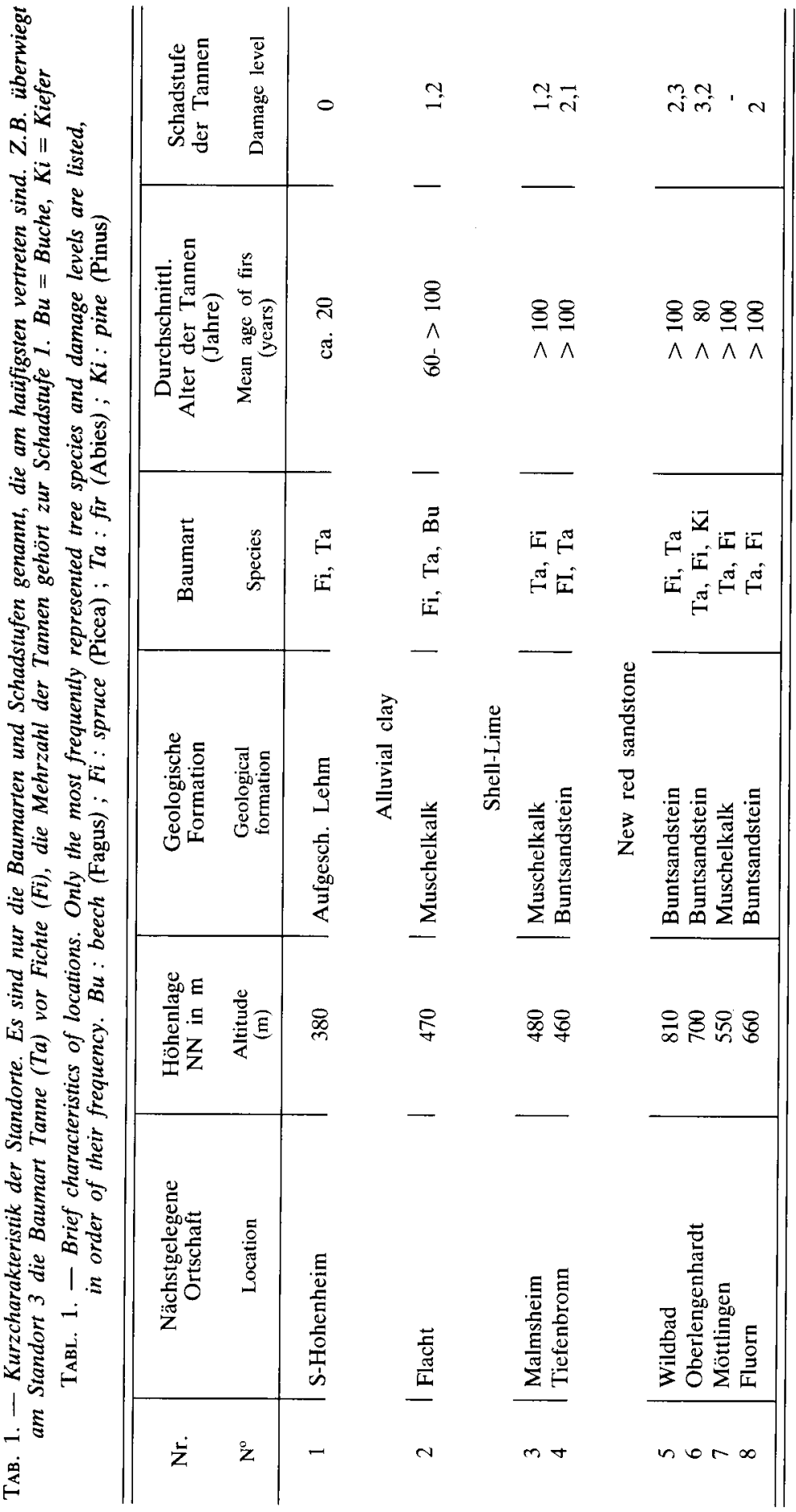


Stellung am Baum bestanden die Zweige aus dem Zuwachs von 2 bis 5 Jahren (bei der Markierung im April). Die markierten Zweige wurden von Mai bis Oktober in wöchentlichen Abständen nach Lachniden abgesucht. Die Stellung der Zweige am Baum bzw. ihre Wüchsigkeit wirken sich nicht auf den Lachnidenbesatz aus. Die Anzahl der auf den Zweigen einer Tanne sitzenden Lachniden wurde addiert ; von dem Lachnidenbesatz der Versuchsbäume wurde der Mittelwert Lachniden je Baum (L/B) gebildet.

An den Standorten 2-8 wurde die Populationsdichte durch Abklopfen der Lachniden von den Zweigen in ein Fangtuch $(60 \times 60 \mathrm{~cm})$ festgestellt. Durch das Abklopfen von 3 entsprechend großen Zweigen erhält man die Anzahl Lachniden je qm Zweigfläche (L/qm). Jeder Standort wurde in 3 Bereiche eingeteilt, in jedem Bereich wurden bei jeder Messung 3 Zweige abgeklopft. Von den 3 Werten wurde der Mittelwert berechnet. Die Standardabweichung beträgt im Durchschnitt $30 \%$ des Mittelwertes.

Es wurden nur Zweige abgeklopft, die vom Erdboden aus oder mit einer $3 \mathrm{~m}$ bzw. $5 \mathrm{~m}$ langen Leiter erreichbar waren. Bei 4-6 kräftigen Schlägen fallen 70-100\% der auf den Zweigen befindlichen Lachniden in das Fangtuch. Ältere Tiere fallen leichter als jüngere Larven, wahrscheinlich spiclt das Gewicht eine Rolle. Bei Regen oder regennassen Zweigen liegt die Fallquote deutlich niedriger, deshalb wurden die Zählungen nur bei trockener Witterung durchgeführt. Zwischen den abgeklopften Zweigen treten immer mehr oder weniger große Unterschiede im Lachnidenbesatz auf ; diese Unterschiede stehen weder mit der Stellung der Zweige (Himmelsrichtung, Höhe) bzw. des Baumes noch mit den Wuchsmerkmalen der Zweige (Wüchsigkeit, Benadelung) in einem Zusammenhang.

Die Zählungen wurden von Mai bis Oktober im Abstand von 2 Wochen durchgeführt.

\section{Messung der Eiablage}

An jedem Standort wurden zwischen Ende November und Anfang Dezember von 10 Tannen ( 3 bzw. 4 je Bereich) Zweige mit einer Stangenschere abgeschnitten und mit einem Zollstock ausgemessen. Die Zweige waren zwischen 0,5 und 1,5 qm groß. Sie wurden Nadel für Nadel auf Eier abgesucht. Je Standort wurde die durchschnittliche Anzahl Eier je qm Zweigfläche berechnet (Ei/qm). Der Eibesatz wurde nur an den Standorten 2-8 und in den Jahren 1979-1984 gemessen.

\section{ERGEBNISSE}

Charakteristisch für die Massenwechselkurven sind die Vermehrungsphase in der Zeit des Austriebes, die zu dem Populationsmaximum im Sommer führt, und der sich anschließende Zusammenbruch der Lachnidenpopulation. Der Anstieg der Populationsdichte während des Austriebes kommt durch die Vermehrungstätigkeit der Stammütter und der $F_{1}$-Generation zustande. Der Zusammenbruch vollzieht sich in der Regel nach der Beendigung des Austriebes während des Auftretens der $F_{2}-$ und $F_{3}$-Generation.

Folgende Abweichungen von diesem Grundschema des Massenwechsels sind möglich :

1. Die Vermehrungsphase endet schon während des Austriebes mit dem Zusammenbruch der $\mathrm{F}_{1}$-Generation.

2. Im August/September setzt eine Spätvermehrung ein, die zu der Ausbildung eines zweiten Populationsgipfels im Herbst führt. Der Zusammenbruch kann dieser Spätvermehrung vorausgehen, er kann aber auch nicht oder nur teilweise stattfinden. 


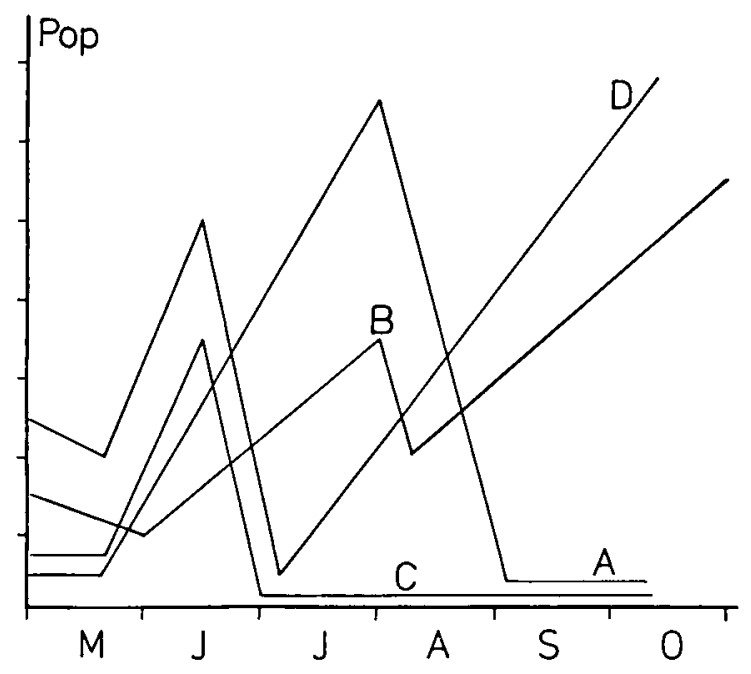

AвB. 1. - Typen des Massenwechsels von C. pectinatae. Dic Unterschiede zwischen den Typen A, B, C und $D$ sind schematisiert und beziehen sich nur auf die Veränderungen der Populationsdichte und nicht auf ihre Höhe. Vgl. auch Abb. 2. Unter der Abszisse stehen die Anfangsbuchstaben der Monate Mai bis

\section{Oktober.}

Typ A : Die Vermehrung von Stammüttern und $F_{1}$ führt zu einem Anstieg in der Populationsdichte im Mai, Juni (und Juli) ; anschlicßend erfolgt der Zusammenbruch im August/September. Die Eiablage ist relativ schlecht.

Typ B : Die Vermehrung von Stammüttern und $F_{1}$ führt wic bci Typ A zu cinem Populationsmaximum im Sommer ; im Unterschied zu Typ A kommt es nach dem Zusammenbruch zu einer Spätvermehrung im August/September und anschließend zu einer relativ guten Eiablage. Der Zusammenbruch ist oft auch nur angedeutet und kann sogar ganz ausbleiben.

Typ C: Während des Austriebes vermehren sich nur dic Stammütter, der Zusammenbruch findet bereits während der $F_{1}$-Generation im Juni statt und führt zu einer niedrigen Populationsdichte im Sommer und im Herbst. Dic Eiablage ist relativ schlecht.

Typ D : Wic bei Typ $C$ vermehren sich nur die Stammütter und die $F_{1}$-Population bricht im Juni zusammen. Es kommt aber anschließend zu einer Spätvermehrung im August/September und damit zu einer relativ guten Eiablage.

FIG. 1. - Types of population trends between May and October. The schematized course of population dynamics shows relative changes, not absolutc values. Pop : population density; abscissa : months MayOctober. See fig. 2.

Type A: Maximum of population causcd by reproduction of fundatrices and $F_{1}$ beginning in May through June (and July), breakdown in August, relatively few eggs in autumn.

Type B : Maximum in summer, second maximum in October, after slight depression.

Type C: Maximum in June, early definite breakdown, few eggs.

Type D : Early maximum in June, breakdown, second maximum until late in autumn with many eggs. 


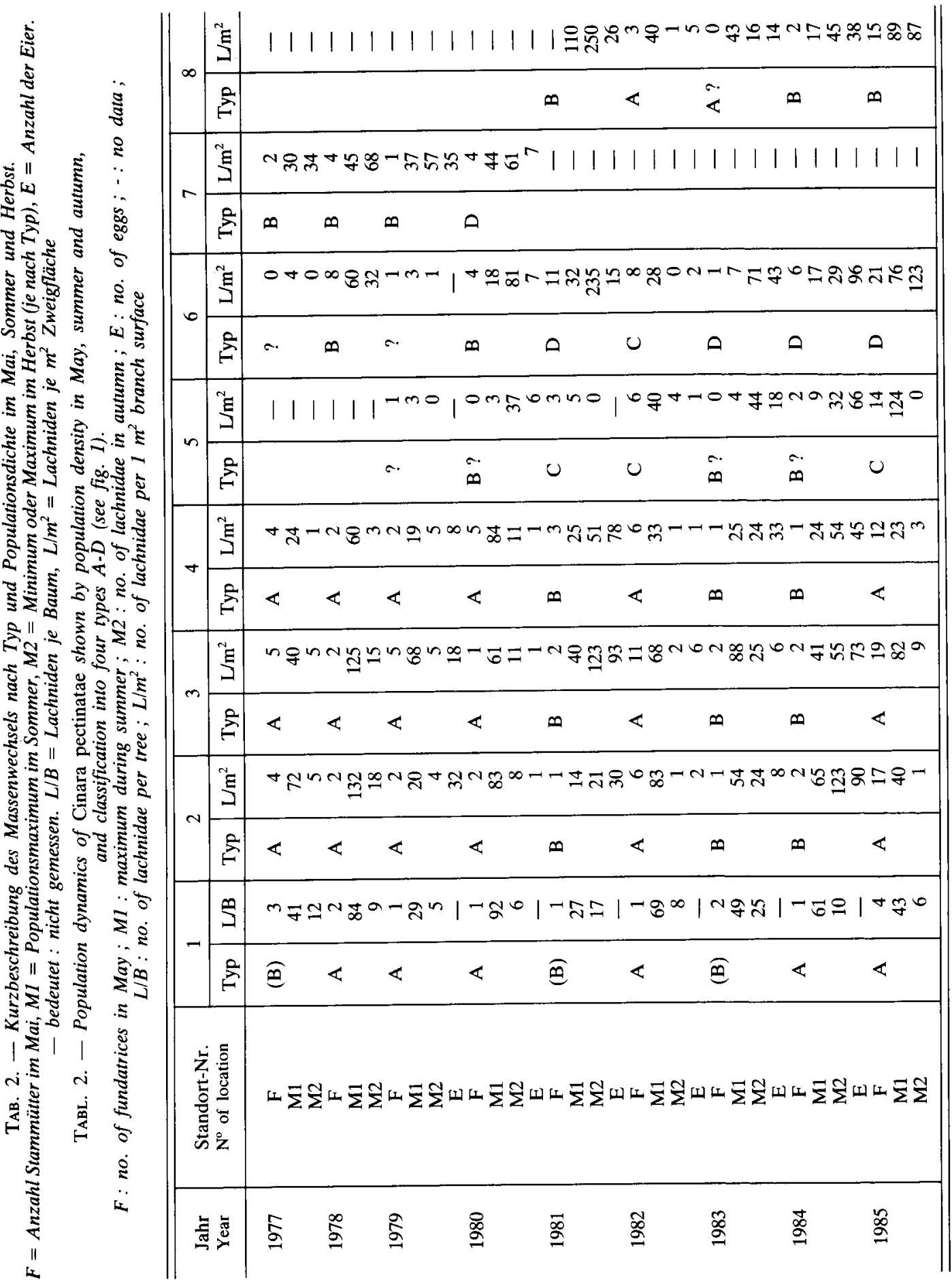


Insgesamt lassen sich so die 61 vorliegenden Massenwechselkurven in 4 Typen (A, B, C und D) einordnen (vgl. Abb. 1).

Die Anzahl der erwachsenen Stammütter zu Beginn der Vermehrungsphase im Mai kann zwischen weniger als 1 und 30 je qm Zweigfläche liegen (vgl. Tab. 2). Das Populationsmaximum im Sommer schwankt zwischen 5 und 150 Lachniden je qm Zweigfläche. Seine Höhe ist nicht mit der Stammutterzahl korreliert, die zu Beginn der Vermehrungsphase festgestellt wurde. Verläuft der Massenwechsel nach Typ A oder C, dann führt der Zusammenbruch zu einer Populationsdichte, die im Herbst in der Mehrzahl der Fälle deutlich unter 20 Lachniden je qm liegt. In der Regel hat dies eine relativ schlechte Eiablage und damit einen relativ niedrigen Stammutterbesatz im Frühjahr des kommenden Jahres zur Folge.

Nach einer Spätvermehrung tritt im September oder Oktober ein zweiter Populationsgipfel auf, der in Hohenheim immer niedriger als das Populationsmaximum im Sommer war. An den Standorten des Schwarzwaldes konnte der Herbstgipfel mit 20-250 L/qm sowohl unter als auch über dem Sommergipfel liegen (vgl. Tab. 2). Nach einer Spätvermehrung wurden in der Regel viele Eier gezählt bzw. ein relativ hoher Stammutterbesatz im Mai des folgenden Jahres festgestellt. Der Eibesatz kann insgesamt zwischen 1 und 100 Eiern je qm Zweigfläche schwanken.

Die Unterschiede, die an einem Standort von Jahr zu Jahr bzw. im gleichen Jahr zwischen den Standorten im Stammutterbesatz, in der Höhe des Populationsmaximums des Sommers, in der Höhe der Populationsdichte im Herbst und im Eibesatz auftreten, können sehr groß sein. Beim Vergleich dieser Werte ist zu erkennen, daß kein Standort für das Auftreten eines Massenbefalls bzw. für sein Ausbleiben prädestiniert ist.

Am Standort 1 wurde die Populationsdichte durch Auszählen der Lachniden auf markierten Zweigen erfaßt. Die Anzahl der Stammütter schwankte von Jahr zu Jahr zwischen 1 und 4 je Baum und war damit relativ einheitlich. 1977 und 1985 wurden die meisten Stammütter gezählt. Der Massenwechsel verlief grundsätzlich nach dem Typ A. Nach den trockenen Sommern 1981 und 1983 sowie im Jahr 1977 trat zwar eine Spätvermehrung auf, die aber immer erst einsetzte, nachdem der Zusammenbruch erfolgt war, und nur zu einem niedrigen, wenn auch deutlich ausgeprägten Populationsgipfel im September führte (vgl. LiEbig u.a., 1982). Das Populationsmaximum des Sommers trat am Standort 1 immer Ende Juni/Anfang Juli auf. 1977, 1979 und 1981 lag es mit durchschnittlich 41, 29 und 27 Lachniden je Baum am niedrigsten. 1982, 1983, 1984 und 1985 schwankte es zwischen 40 und $60 \mathrm{~L} / \mathrm{B}$, die höchsten Werte wurden 1978 und 1980 mit durchschnittlich 84 bzw. 92 L/B gemessen. An den Standorten 2-8 wurde der Lachnidenbesatz durch Abklopfen ermittelt. 
Am Standort 2 (Muschelkalk) verlief der Massenwechsel sechsmal nach dem Typ A, dreimal nach dem Typ B. Im Mai wurden zwischen 1 (1981, 1983) und 17 (1985) Stammütter je qm Zweigfläche gezählt. Die Stammutterzahl war 1985 mit Abstand die höchste, dennoch lag das Populationsmaximum im Sommer lediglich bei 40 Lachniden je qm $(\mathrm{L} / \mathrm{qm})$. Besonders niedrig war dieses außerdem in den Jahren 1979 und 1981 mit 20 bzw. 14 L/qm. 1977, 1980, 1982, 1983 und 1984 lag der Populationsgipfel des Sommers relativ einheitlich hoch mit Werten zwischen 54 und $83 \mathrm{~L} / \mathrm{qm}$. Herausragend war das Jahr 1978 mit $132 \mathrm{~L} / \mathrm{qm}$ im August (vgl. auch Abb. 2). Eine Spätvermehrung wurde 1981, 1983 und 1984 festgestellt. Dabei stieg der Lachnidenbesatz 1981 und $1983 \mathrm{im}$ September lediglich auf $21 \mathrm{bzw} .24 \mathrm{~L} / \mathrm{qm}, 1984$ dagegen auf $123 \mathrm{~L} / \mathrm{qm}$ an, was auch eine entsprechend hohe Eiablage und damit im folgenden Jahr 1985 den mit Abstand höchsten Stammutterbesatz des Zeitraumes 1977-1985 zur Folge hatte.

Der Massenwechsel am Standort 3 (Muschelkalk) ist dem des Standortes 2 sehr ähnlich (vgl. Tab. 2), der Massenwechsel-Typ ist jedes Jahr an beiden Standorten derselbe. Allerdings wurden in einzelnen Jahren sehr große Unterschiede im Populationsmaximum im Sommer bzw. im Herbst festgestellt. Der Populationsgipfel im Sommer lag zwischen 40 und $125 \mathrm{~L} / \mathrm{qm}$, wobei das Jahr 1978 wie bei Standort 2 herausragt. Die niedrigsten Maxima traten 1977, 1981 und 1984 auf mit 40 bzw. 41 L/qm. 1979, 1980, 1982, 1983 und 1985 war das sommerliche Maximum mit 60-88 L/qm relativ einheitlich hoch. Eine Spätvermehrung wurde wie am Standort 2 in den Jahren 1981, 1983 und 1984 festgestellt, die 1981 und 1984 zu einem Herbstmaximum führte, bei dem mit $125 \mathrm{~L} / q m$ bzw. $55 \mathrm{~L} /$ qm mehr Lachniden gezählt wurden als im Sommer; 1983 waren es dagegen mit $25 \mathrm{~L} / \mathrm{qm}$ deutlich weniger.

Der Massenwechsel am Standort 4 (Buntsandstein) ähnelte vom Typ her dem der Standorte 2 und 3 (vgl. Tab. 2). Der Stammutterbesatz lag im Mai zwischen 1 und $12 \mathrm{~L} / \mathrm{qm}$. Die meisten Stammütter wurden wie an den Standorten 2 und 3 im Mai 1985 gezählt. Im Bezug auf das Populationsmaximum des Sommers ragen 2 Jahre heraus : 1978 waren es $60 \mathrm{~L} / \mathrm{qm}, 1980$ sogar $84 \mathrm{~L} /$ qm. In den übrigen Jahren lag der Sommergipfel zwischen 19 und $33 \mathrm{~L} / \mathrm{qm}$. Eine Spätvermehrung wurde wie an den Standorten 2 und 3 in den Jahren 1981, 1983 und 1984 beobachtet. Das Maximum im Herbst betrug 1983 lediglich $24 \mathrm{~L} / \mathrm{qm}, 1981$ und 1984 waren es 51 bzw. 54 L/qm.

Am Standort 5 (Buntsandstein) wurde erst 1979 mit den Messungen begonnen. Während an den Standorten 1, 2, 3 und 4 der Massenwechseltyp A vorherrscht und als Variante lediglich Typ B (= A mit Spätvermehrung) auftritt, verlief der Massenwechsel am Standort 5 entweder nach dem Typ B oder nach dem Typ C. Die Stammutterzahl schwankte 1979-1984 zwischen 0 und 5 je qm, 1985 wurden im Mai 14 Stammütter je qm gezählt. 1981, 1982 

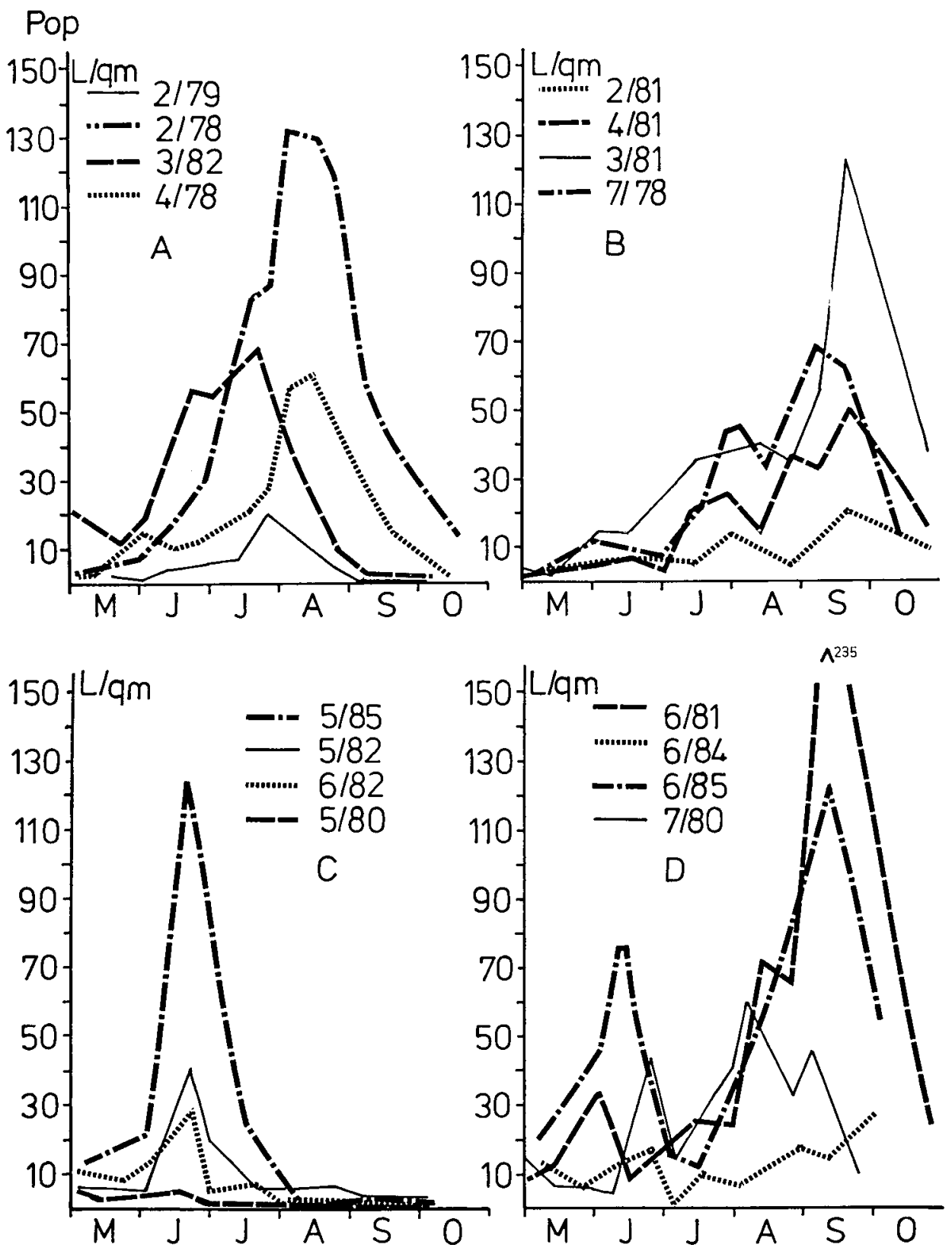

Aвв. 2. - Der Massenwechsel von C. pectinatae an verschiedenen Standorten.

Beispiele für die Massenwechseltypen A, B, C und D. Die Zahl vor dem Schrägstrich gibt den Standort an, die dahinter die die Jahreszahl. L/qm = Lachniden je qm Zweigfläche.

FIG. 2. - Individual population development during the season in different locations and years, arranged according to type of population dynamics (A-D ; see fig. 1).

Pop : population density ; L/qm : no. of Lachnidae per $\mathrm{m}^{2}$; first figure : no. of location ; second figure : year. 
und 1985 brach die Population bereits mit dem Auftreten der $F_{1}$-Generation zusammen, d.h. das Maximum wurde bereits Mitte Juni gemessen mit 5 (1981), 40 (1982) bzw. 124 L/qm (1985). 1979, 1980, 1983 und 1984 vermehrten sich Stammütter und $F_{1}$ nur sehr schwach, so daß in diesen Jahren das sommerliche Populationsmaximum unter $10 \mathrm{~L} / \mathrm{qm}$ lag. Die Spätvermehrung 1980, 1983 und 1984 führte zu einem Maximum im September, das mit Werten zwischen 32 und $44 \mathrm{~L} / \mathrm{qm}$ deutlich höher lag als das jeweilige Maximum des Sommers.

Der Standort 6 (Buntsandstein) zeichnet sich dadurch aus, daß der Zusammenbruch der $F_{1}$-Generation und die Spätvermehrung sehr häufig auftraten (5bzw. 6-mal). 1977 und 1979 lag der Lachnidenbesatz bei allen Messungen unter $5 \mathrm{~L} / \mathrm{qm}$, so daß eine Typen-Bezeichnung des Massenwechsels in diesen Jahren nicht möglich ist. Mit Ausnahme des Jahres 1982 fand in den übrigen Jahren eine Spätvermehrung statt, die immer zu einem deutlichen Anstieg der Populationsdichte im September/Oktober führte, wenn auch das herbstliche Maximum von Jahr zu Jahr stark schwankte (29 - 235 L/qm). Die Vermehrungsrate der Stammütter lag in den Jahren 1981-1985 etwa bei dem Faktor 3, je nach Stammutterzahl wurde im Juni ein Maximum der $F_{1}$-Population zwischen 7 und $76 \mathrm{~L} / \mathrm{qm}$ festgestellt. Der Zusammenbruch der $F_{1}$-Generation vollzog sich jedes Jahr innerhalb von 2 Wochen (vgl. Abb. 2). 1981 ging die $F_{1}$-Population von 32 auf $8 \mathrm{~L} / \mathrm{qm}$ zurück, 1982 von 28 auf 6,1983 von 7 auf 2,1984 von 17 auf 1 und 1985 von 76 auf $15 \mathrm{~L} / \mathrm{qm}$.

Am Standort 7 (Muschelkalk) wurde nur in den Jahren 1977-1980 gemessen. Für diesen Standort war typisch, daß jedes Jahr eine Spätvermehrung beobachtet wurde. Die von Jahr zu Jahr auftretenden Unterschiede im Stammutterbesatz und in der Höhe des sommerlichen bzw. herbstlichen Populationsgipfels waren verglichen mit den anderen Standorten sehr gering. Der Stammutterbesatz schwankte zwischen 1 und 4, das Populationsmaximum des Sommers zwischen 30 und 45 und das Populationsmaximum des Herbstes zwischen 34 und $68 \mathrm{~L} / \mathrm{qm}$.

Der Standort 7 wurde 1981 aufgegeben und gegen den Standort 8 (Buntsandstein) getauscht. Standort 8 war eine sogenannte Trachtinsel des Jahres 1981, im Juli wurde ein Lachnidenbesatz von mehr als $100 \mathrm{~L} / \mathrm{qm}$ gemessen und damit deutlich mehr als im gleichen Zeitraum an den Standorten 2-6. Im August und September 1981 wurde wie an den anderen Standorten (mit Ausnahme des Standortes 5) eine Spätvermehrung festgestellt, die zu einem Populationsmaximum von $250 \mathrm{~L} / \mathrm{qm}$ Ende September führte. Der Stammutterbesatz lag 1982-1984 immer unter $4 \mathrm{~L} / \mathrm{qm}, 1985$ wurden 15 Stammütter je qm gezählt. Das Populationsmaximum des Sommers lag 1982-1985 zwischen 17 und $89 \mathrm{~L} / \mathrm{qm} .1984$ und 1985 setzte wie 1981 im August im Anschluß an den Zusammenbruch eine deutliche Spätvermehrung ein, die ein Herbstmaximum von 45 bzw. $87 \mathrm{~L} / \mathrm{qm}$ zur Folge hatte. 


\section{DISKUSSION}

Der Massenwechsel von Aphiden wird allgemein mit Schwankungen im Gehalt des Phloemsaftes an löslichen Stickstoffverbindungen erklärt (OHNESORGE, 1976). Auch bei $C$. pectinatae sind Fertilität und Mortalität in erster Linie eine Frage ihrer Ernährung. Nach Maquelin (1974) und eigenen Beobachtungen haben Prädatoren, Parasiten und Krankheiten keinen wesentlichen Einfluß auf den Massenwechsel von $C$. pectinatae. Der direkte Vergleich des Massenwechsels mit dem Witterungsverlauf zeigt, daß die Veränderungen in der Populationsdichte nicht durch Temperaturschwankungen und Niederschläge verursacht werden (Liebig u.a., 1982) : die von Jahr zu Jahr unterschiedlich starke Vermehrungstätigkeit von Stammüttern und $F_{1}$ steht ebenfalls in keinem erkennbaren Zusammenhang zum Witterungsverlauf des Frühjahrs bzw. des Frühsommers.

Der Massenwechsel ist wahrscheinlich in erster Linie von der Zusammensetzung des Phloemsaftes abhängig, die sich während der Vegetationszeit ändert und außerdem unter dem Einfluß von Standort- und Witterungsfaktoren steht. Bei Blattgehölzen ist der Stickstoffgehalt des Phloemsaftes besonders hoch während des Austriebes und vor dem Blattfall. Entsprechend ist ein Populationsmaximum nach Beendigung des Austriebes und ein weiteres vor Beginn des Blattfalls möglich; eine zweigipflige Populationskurve wird daher bei vielen auf Blattgehölzen lebenden Blattlausarten als charakteristisch betrachtet (Kloft, 1960 ; Ohnesorge, 1976 ; Kloft und Kunkel, 1985), was aber nicht immer zutrifft (Неimbach, 1984).

Ähnliche Verhältnisse liegen wahrscheinlich auch bei Koniferen vor, da bei den vorliegenden Untersuchungen über $C$. pectinatae neben dem Populationsmaximum im Sommer (während bzw. nach Beendigung des Austriebes) ein weiteres im Herbst auftreten kann, das aber oft gefehlt hat. Bei Fichtenlachniden wurde ebenfalls in der Regel nur ein Populationsmaximum festgestellt (z.B. Thalenhorst, 1972; Eckloff, 1972), lediglich Scheurer (1964) hatte im September ein zweites neben dem im Juni beobachtet.

Untersuchungen über die Intensität und Dauer des Längenwachstums sowie über die Veränderung des Nährelementgehaltes in den Knospen bzw. den Maitrieben während der Vegetationszeit lassen u.a. den Schluß zu, daß von April bis Oktober ein ständiger akropetaler N-Transport stattfindet. Dabei treten bei der Transportleistung jedes Jahr zwei Maxima auf : ein größeres im Mai/Juni zur Deckung des Nährstoffbedarfs der wachsenden Maitriebe und ein kleineres im August/September bei der Einlagerung von Nährstoffen in den Knospen bzw. als Reserven in die dies- und mehrjährigen Triebe (LiebiG, in Vorbereitung). Da sich die von Jahr zu Jahr auftretenden Unterschiede im 
Massenwechsel nicht damit erklären lassen, daß die Höhe der Populationsmaxima von der Transportleistung an N-Verbindungen abhängig ist, haben wir die Arbeitshypothese abgeleitet, daß der akropetale N-Transport sowohl im Phloem als auch im Xylem stattfindet und die Fertilität von $C$. pectinatae sowohl während des Austriebes im Frühjahr und Frühsommer als auch während der Nährstoffeinlagerung im Spätsommer in erster Linie davon abhängt, ob und wieviel N-Verbindungen im Phloem transportiert werden.

Der Massenwechsel von $C$. pectinatae verläuft in der Regel nach Typ A, bei dem sich die Lachniden nur in der Zeit des Austriebes vermehren. In diesem Fall ist der N-Transport im Phloem auf die Zeit des Austriebes begrenzt. Spätestens nach Abschluß des Längenwachstums produzieren die Nadeln einen Überschuß an Assimilaten, die im Phloem abgeleitet werden. Es findet zwar weiterhin ein akropetaler Transport von Nährstoffen (u.a. auch von Stickstoff) statt, was nach unseren Vermutungen bei guten Assimilationsbedingungen aber ausschließlich im Xylem geschieht, wo die $\mathrm{N}-$ Verbindungen von den Lachniden nicht erreicht werden können. (Auf den Transport von Aminosäuren im Xylem hat bereits Bollard (1960) aufmerksam gemacht). Der Phloemsaft enthält dann wenig $\mathbf{N}$ und viel Zucker, entsprechend niedrig ist die Fertilität der Lachniden nach Beendigung des Austriebes. Die von Jahr zu Jahr bzw. von Standort zu Standort unterschiedlich starke Vermehrungstätigkeit während des Austriebes ist demnach wahrscheinlich mit einer witterungs- bzw. standortbedingt unterschiedlich hohen Assimilationsleistung zu erklären : je früher ein Assimilatüberschuß erzeugt wird, desto niedriger ist die Vermehrungsrate von Stammüttern und $F_{1}$ bzw. desto kürzer dauert die Vermehrungsphase.

An den Standorten 1-4 verlief der Massenwechsel in der Mehrzahl der Jahre nach dem Typ A. Am Standort 1 wurde der Massenwechsel an jungen, gesunden Tannen verfolgt. An diesem Standort kam es jedes Jahr nach der Vermehrungsphase zum Zusammenbruch der Lachnidenpopulation. Eine anschließende Spätvermehrung wurde nur 1977, 1981 und 1983 festgestellt, war aber immer nur schwach ausgeprägt. An den Standorten 2, 3 und 4 trat die Spätvermehrung ebenfalls 1981 und 1983, im Unterschied zu Standort 1 aber auch 1984, nicht dagegen 1977 auf.

MaqueulN (1974) stellte bei seinen Untersuchungen über den Massenwechsel von C. pectinatae von 1966-1972 in Tannenbeständen der Schweiz wiederholt eine Spätvermehrung fest. Er vermutet, daß Standortfaktoren wie Bodenverhältnisse und die Wasserversorgung einen entscheidenden Einfluß auf die Populationsdynamik ausüben; allerdings begründet MAQUELIN diese Aussage nicht näher.

Die Spätvermehrung der Jahre 1981 und 1983 hängt wahrscheinlich damit zusammen, daß der Sommer in diesen Jahren sehr trocken war. Bei anhaltend 
schlechter Wasserversorgung sinkt die Assimilationsleistung der Tannen und damit wahrscheinlich auch die Transportleistung des Xylems. Das Auftreten der Spätvermehrung wäre dann damit zu erklären, daß der akropetale Nährstofftransport im August/September bei Wassermangel im Phloem durchgeführt wird, was ein Ansteigen des $\mathrm{N}$-Gehaltes im Phloemsaft und damit eine Erhöhung seines Nährwertes zur Folge hätte. Eine entsprechende Erklärung könnte auch auf den Standort 7 zutreffen, an dem der Massenwechsel jedes Jahr nach dem Typ B verlief. Bei diesem Standort handelt es sich um eine trockene Muschelkalkkuppe, auf der für Tannen sehr ungünstige Wuchsbedingungen bestehen.

Insgesamt ziehen wir daraus die Schlußfolgerung, daß eine Spätvermehrung immer dann auftritt, wenn witterungs- oder standortbedingt schlechte Assimilationsbedingungen herrschen. Dies kann durch Trockenheit oder auch durch die Tannenerkrankung verursacht werden, die am Standort 6, an dem die Spätvermehrung am häufigsten beobachtet wurde, besonders weit fortgeschritten ist. Vielleicht liegt hier eine pathologisch bedingte Störung des Wasserhaushaltes vor, wie sie auch bei stark erkrankten Fichten vermutet wird (Strack und Unger, 1986), die es notwendig macht, daß der akropetale Nährstofftransport mindestens zum Teil im Phloem durchgeführt wird.

Für den Standort 6 ist weiterhin auffällig, daß seit 1981 regelmäßig der Zusammenbruch der $F_{1}$-Population schon während des Austriebes stattfindet. Zur gleichen Zeit dauert die Vermehrungsphase an anderen Standorten noch an. Dieser verfrühte Zusammenbruch steht eventuell ebenfalls mit der Tannenerkrankung in einem Zusammenhang. In Anlehnung an unsere Arbeitshypothese kann man vermuten, daß bei stark erkrankten Tannen der N-Gehalt des Phloemsaftes schon frühzeitig abnimmt, weil schon in der Anfangsphase des Austriebes ein intensiver basipetaler Assimilattransport z.B. für die laufende Erneuerung geschädigter Wurzeln durchgeführt wird. So haben OBERwinKLER u.a. (1986) bei einer stark erkrankten Tanne mehr Feinstwurzeln gefunden als bei einer gesunden Tanne. Bei der erkrankten Tanne war die Wurzelaktivität gerade im Juni besonders stark erhöht. Allerdings können diese Ergebnisse nicht verallgemeinert werden, da sie nur an einem Baumpaar während eines Jahres erzielt wurden.

Die Beobachtung der Populationsdynamik von $C$. pectinatae während des Zeitraumes von 1977 bis 1985 läßt nicht erkennen, daß es in erkrankten Tannenbeständen häufiger oder seltener zu einer Massenvermehrung kommt. Allerdings zeichnet sich ab, daß ein Massenbefall, wenn es zu einem solchen kommt, in stark erkrankten Beständen entweder früher (im Juni) oder später (im September) auftritt. In beiden Fällen kann eine einsetzende Tannentracht nicht oder nur bedingt genutzt werden. In der Regel verfügt der Imker im Juni noch nicht über leistungsstarke Bienenvölker, um eine früh einsetzende Tan- 
nentracht anzuwandern. Die Nutzung einer späten Tannentracht ist besonders für die in Tannentrachtgebieten ansässigen Standortimker problematisch, da sie die Überwinterungsfähigkeit der Bienenvölker stark gefährdet. Außerdem nimmt ab August die Volksstärke natürlicherweise ab, so daß eine Tannentracht im September nur bei sehr günstiger Witterung zufriedenstellende Honigerträge bringt.

Eingegangen im April 1986 Angenommen im November 1986.

\title{
DANKSAGUNG
}

Dic Untersuchungen werden von der DFG finanziell unterstützt. ich danke Frau Ursula Schlebusch für ihre langjährige Mitarbeit bei der Durchführung der Messungen.

\author{
RÉSUMÉ \\ LA DYNAMIQUE DES POPULATIONS DU PUCERON CINARA PECTINATAE \\ DANS DES SAPINIĖRES DIVERSEMENT MALADES, ENTRE 1977 ET 1985
}

Pour les apiculteurs du sud de l'Allemagne, la forêt représente la principale source de miellée. Cinara pectinatae est le puceron producteur de miellat le plus important. Il se nourrit sur le sapin argenté Abies alba.

On a ćtudié sa dynamique de populations depuis 1977 en 7 cndroits du Bade-Württemberg (Tabl. 1). Le but était de déterminer les conditions et les obstacles à une miellée sur le sapin argenté. Les plantations observées étaient malades à des degrés divers; on a donc contrôlé si la maladie des sapins avait une influence sur la dynamique des populations de $C$. pectinatae.

La densité de population a été mesuréc tous les 15 jours de mai à octobre : on frappait les branches pour faire tomber les pucerons puis les récolter ou bien on les comptait sur des branches marquées. A la fin de l'automne on comptait les œufs.

Le schéma typique de population comprend une phase de multiplication au moment de la pousse des arbres en mai-juin-juillet, puis un effondrement en août et scptembre (Fig. 1 et 2).

L'activité de multiplication des fondatrices et de la génération $F_{1}$ provoque un pic de densité de population, qui s'observe généralement en juillet/août. Il varie selon les annécs et les endroits : on a observé moins de 10 ou plus de 100 pucerons sur unc surface de branches d'1 $\mathrm{m}^{2}$ (Tabl. 2).

Dans les sapinières très malades, il y a un second pic en septembre, causé par la multiplication intensive des pucerons à la fin de l'été (Fig. 1 et 2). En général cette multiplication intensive apparaît principalement après un été sec (Tabl. 2). Si lc temps est favorable, il y a parfois une miellée tardive en septcmbre mais il est difficilc pour les apiculteurs d'en tirer profit.

Due également à la multiplication tardive, unc pontc intense a lieu en automne et donc une densité élevée de fondatrices l'année suivante en mai (Tabl. 2). En dépit de ces conditions favorables au début de l'année suivante, il n'y a pas de micllée en juillet/août dans les forêts de sapins fortement malades, parce que la population de la génération $\mathrm{F}_{1}$ s'effondre en juin au moment de la pousse des arbres. 


\section{SUMMARY \\ POPULATION DYNAMICS OF THE GREEN STRIPED FIR APHID CINARA PECTINATAE IN VARIOUSLY DISEASED FIR STANDS, 1977-1985}

For the beekeepers in Southern Germany the forest is the main source of honey flow. Cinara pectinatae is the most important honeydew-producing aphid feeding on white fir, Abies alba.

Since 1977, at 7 places in Baden-Württemberg (Tabl. 1), investigations have been made on its population dynamics. The goal was to determine the conditions and the hinderances for a honey flow from white fir. The observed fir plantations were variously diseased; therefore it was checked whether the disease of firs had an influence on the population dynamics of $C$. pectinatae.

The population density was measured from May to October fortnightly, the lachnids were beaten off branches and collected or were counted on marked branches. In late autumn the number of eggs was recorded.

Typical population trends included a multiplication process during sprouting of trees in May, June and July, and a collapse of the populations afterwards in August and September (Fig. 1, 2).

The multiplication activity of fundatrices and the $\mathrm{F}_{1}$-generation caused a peak of the population density, observed in July/August. In different years and at different places there were fewer than 10 or more than 100 lachnids on branches of square meter in size (Tabl. 2).

In heavily diseased fir plantations there was a second peak of population density in September, caused by an intensive multiplication of the lachnids in the late summer (Fig. 1, 2). In general this late multiplication was especially apparent after a dry summer (Tabl. 2). If the weather was favorable there was sometimes late honey flow in September. However, for beekeepers it is difficult to make some profit on it.

Also due to the late multiplication there was an intensive oviposition in autumn and a high density of fundatrices in the following year in May (Tabl. 2). In spite of favorable conditions at the beginning of the next year there was not a honey flow of heavily diseased white fir forests in July/August because the population of the $F_{1}$-generation collapsed in June during sprouting of trees.

\section{LITERATUR}

Bollard E.G., 1960. - Transport in the xylem. Annu. Rev. Plant Physiol., 11, 141-166.

ECKLOFF W., 1972. - Beitrag zur Ökologie und forstlichen Bedeutung bienenwirtschaftlich wichtiger Rindenläuse. Z. ang. Entomol., 70, 134-157.

Негмвасн U., 1984. - Freilanduntersuchungen zur Honigtauausscheidung und Populationsdynamik zweier Zierlausarten (Aphidina) an Linden und Eichen im Hinblick auf die Honigtaunutzung durch Honigbienen. Dissertation, Hannover.

Kloft W., 1960. - Die Honigtauerzeuger. In : Büdel A., Herold H., Biene und Bienenzucht. München, Ehrenwirth Verlag, 105-114.

KLoFT W.J. und Kunkel H., 1985. - Waldtracht und Waldhonig in der Imkerei, Ehrenwirth-Verlag, München.

Liebig G., Schlipf U. und Düwel W., 1982. - Witterungsverlauf und Massenwechsel der Grünen Tannenhoniglaus Cinara pectinatae (Nördl.) (Homoptera, Lachnidae) in den Jahren 1977-1981. Apidologie, 13 (3), 275-295.

LIEBIG G. (in Vorbereitung). - Der Vergleich des Massenwechsels der Grünen Tannenhoniglaus Cinara pectinatae mit der Nährstoffversorgung ihres Wirtsbaumes. 
Maquelin Ch., 1974. - Observations sur la biologie et l'écologie d'un puceron utile à l'apiculture : Buchneria pectinatae (Nördl.) (Homoptera, Lachnidae). Dissertation, Zürich.

Oberwinkler F., Kottke I., Ritter Th. und Feil W., 1986. - Vergleichende Untersuchungen der Fcinstwurzelsysteme und der Anatomic von Mykorrhizen nach Trockenstre $B$ und Düngemaßnahmen. Projekt Europ. Forschungszentrum für Maßnahmen zur Luftreinhaltung (PEF), PEF-Berichte, 4 (1), 315-331.

Ohnesorge B., 1976. - Tiere als Pflanzenschädlinge, Thieme-Verlag, Stuttgart.

Scheurer S., 1964. - Generationenfolge und Aufenthaltsorte der im östlichen Teil des Harzes gefundenen Lachniden. Disch. Bienenwirtsch., 15, 214-217.

SCHÖPfER W. und Hradetzky J., 1985. - Ergebnisse der ersten Waldschadens-Wiederholungsinventur in Baden-Württemberg. In : BAuer F., Die Sache mit dem Wald. - BLV-Verlagsgesellschaft MünchenWien-Zürich, 93-98.

Schröter H. und Aldinger E., 1985. - Beurteilung des Gesundheitszustandes von Fichte und Tanne nach der Benadelungsdichte. In : Bauer F., Die Sache mit dem Wald. - BLV-Verlagsgesellschaft München-Wien-Zürich, 122-125.

STrack S. und UNGer H., 1986. - Untersuchungen des Wassertransports und der Wasserumsätze in Fichten mit Hilfe von Tritium. Projekt Europ. Forschungszentrum für Maßnahmen zur Luftreinhaltung (PEF), PEF-Berichte, 4 (1), 213-225.

Thalenhorst W., 1972. - Düngung, Wuchsmerkmale der Fichte und Arthropodenbefall. Mitt. Niedersächs. Landesforstverwaltung, 18. 